\title{
Caracterización epidemiológica de las infecciones de vías urinarias asociadas al cuidado de la salud en la Unidad de Cuidados Intensivos Neonatales
}

\author{
Epidemiological characterization of healthcare-associated urinary \\ tract infections in the Neonatal Intensive Care Unit
}

\author{
Adriana Nieto Sanjuanero, ${ }^{*}$ Ma. de Jesús Sotelo Gallegos, ${ }^{*}$ Abiel Homero Mascareñas de los Santos, ${ }^{\ddagger}$ \\ José Iván Castillo Bejarano, ${ }^{\ddagger}$ Lorena Salazar Cavazos, ${ }^{\S}$ Carlos De la Cruz De la Cruz, ${ }^{*}$ \\ Mónica Janeth Olivares Ramírez* \\ * Unidad de Cuidados Intensivos Neonatales, Departamento de Pediatría, Servicio de Neonatología. \\ ‡ Departamento de Pediatría, Servicio de Infectología. \\ § Departamento de Patología Clínica, Servicio de Bacteriología.
}

Hospital Universitario «Dr. José Eleuterio González», Universidad Autónoma de Nuevo León. Monterrey, Nuevo León, México.

\section{RESUMEN}

Introducción: En la Unidad de Cuidados Intensivos Neonatales (UCIN), el riesgo de infección está relacionado con la duración de la hospitalización y la inmadurez del sistema inmunológico de los recién nacidos. Dentro de las infecciones asociadas con los cuidados de la salud, las infecciones de vías urinarias son las más frecuentes. Objetivo: Determinar el rendimiento diagnóstico del examen general de orina (EGO) frente al urocultivo en IVU de recién nacidos hospitalizados en una Unidad de Cuidados Intensivos Neonatales (UCIN). Material y métodos: Estudio observacional y retrospectivo. Se incluyeron neonatos con sospecha clínica de IVU ingresados en una UCIN de enero de 2018 a junio de 2019. Se incluyeron neonatos menores a 30 días de vida. Se documentaron características clínicas, parámetros bioquímicos del EGO y se compararon con el perfil bacteriológico del urocultivo. Para evaluar el rendimiento se realizó un análisis estadístico por medio de tablas tetracóricas, se consideró una $\mathrm{p}<$ 0.05 como estadísticamente significativo. Resultados: Se incluyeron 172 pacientes con sospecha de IVU, 32 (18.6\%) con urocultivo positivo. La edad gestacional del grupo con urocultivo positivo fue $33 \pm 4.5$ semanas de gestación vs $34.6 \pm 3.6$ del grupo con urocultivos negativos $(p=0.034)$. El microorganismo más frecuente fue Klebsiella pneumoniae (31.5\%), seguido de Escherichia coli (26.5\%). La falla de medro fue la manifestación clínica más común. La sensibilidad fue $<50 \%$ en todos los parámetros del examen general de orina. Encontramos especificidad de $95.7 \%$ para nitritos

Financiamiento: Ninguno.

Conflicto de intereses: Ninguno.

Rev Latin Infect Pediatr. 2021; 34 (1): 22-26

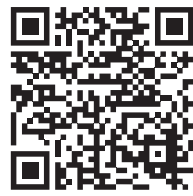

\section{ABSTRACT}

Introduction: In the Neonatal Intensive Care Unit (NICU), the risk of infection is related to the length of hospitalization and the immaturity of the immune system of newborns. Among healthcareassociated infections, urinary tract infections are the most frequent. Objective: To determine the diagnostic performance of the urine general examination (UGE) versus urine culture in UTI in newborns hospitalized in a neonatal intensive care unit (NICU). Material and methods: Observational and retrospective study. We included laboratory reports of consecutive newborns with clinical suspicion of UTI admitted in a NICU from January 2018 to June 2019. We recollected clinical urine tests and cultures parameters from the same sample and assessed the diagnostic yield of some of the urianalysis parameters associated to the presence of infection. We also reported some clinical manifestations presented by patients. Results: We included 172 patients with suspicion of UTI, 32 (18.6\%) presented a positive urine culture. The gestational age of the positive urine culture group was lower, $33 \pm 4.5$ weeks of gestation (WG) vs $34.6 \pm$ 3.6 WG in the negative urine culture patients $(p=0.034)$. The most common isolated bacteria were Klebsiella pneumoniae (31.5\%), followed by Escherichia coli (26.5\%). Failure to thrive was the most frequent clinical manifestation $(p=0.006)$. We did not find a sensibility higher than $50 \%$ in any individual or combined urianalysis parameter for positive urine cultures. We found a specificity of $95.7 \%$ for nitrites and $98.6 \%$ for yeast.

Citar como: Nieto SA, Sotelo GMJ, Mascareñas SAH, Castillo BJI, Salazar CL, De la Cruz CC et al. Caracterización epidemiológica de las infecciones de vías urinarias asociadas al cuidado de la salud en la Unidad de Cuidados Intensivos Neonatales. Rev Latin Infect Pediatr. 2021; 34 (1): 22-26. https:// dx.doi.org/10.35366/99824 
Rev Latin Infect Pediatr. 2021; 34 (1): 22-26

y cultivo positivo y en infecciones fúngicas una especificidad de $98.6 \%$ en levaduras y urocultivo positivo. Conclusiones: No encontramos un buen rendimiento diagnóstico en los parámetros del urianálisis aislados o en combinación con el urocultivo positivo para la identificación de infecciones urinarias asociadas con los cuidados de la salud en recién nacidos internados en la UCIN. Debemos considerar la falla de medro como manifestación clínica de infección urinaria más frecuente en esta población.

Palabras clave: Infección vías urinarias neonatal, perfil bacteriológico, infecciones urinarias nosocomiales.

\section{INTRODUCCIÓN}

Las infecciones de vías urinarias (IVU) son entidades frecuentes en población pediátrica. La prevalencia de IVU se reporta en alrededor de 0.1 a $1 \%$, y aumenta hasta $25 \%$ en recién nacidos prematuros. ${ }^{1,2} \mathrm{La}$ infección por vía hematógena es común en neonatos, la mayoría comienza en hígado, y posteriormente se disemina a vía urinaria, hasta producir una bacteriemia. $^{3}$

Algunos factores de riesgo relacionados con el desarrollo de IVU asociada con los cuidados de la salud en recién nacidos son: género masculino, bajo peso al nacer, la ausencia de circuncisión, uso de antibióticos de amplio espectro, malformaciones renales, ventilación mecánica, uso prolongado de sondas y catéteres y prematuros menores de 36 semanas de gestación. , $^{1,5}$ En la Unidad de Cuidados Intensivos Neonatales (UCIN), el riesgo de infección se relaciona con la estancia hospitalaria y la inmadurez del sistema inmunológico de los recién nacidos. ${ }^{6,7}$

Algunos patógenos frecuentemente son bacilos Gram negativos, como Escherichia coli, Klebsiella spp., y Enterobacter spp., además de agentes como Candida spp. 2,8,9

La presentación clínica en el neonato es inespecífica, pero se pueden encontrar datos de intolerancia a la vía oral, falla de medro, letargo, vómito, diarrea, irritabilidad, fiebre o hipotermia, hipoglucemia, distensión abdominal, bradicardia e ictericia prolongada. . $^{6,10}$

La presencia de una IVU puede ser un dato de alarma de anormalidades funcionales o anatómicas del riñón y/o tracto urinario. ${ }^{3}$

El estándar de oro para la confirmación de IVU es el urocultivo, considerado positivo cuando se reporta un umbral igual o mayor a 50,000 UFC/ $\mathrm{mL}$ de un solo organismo, o un cultivo tomado por cateterización estéril con un resultado igual o mayor a $10,000 \mathrm{UFC} / \mathrm{mL}$ con un solo organismo, acompañado de piuria. ${ }^{11}$ Definiendo piuria al recuento igual
Conclusions: We did not find a good diagnostic yield within individual or combined urianalysis parameters for positive urine cultures in the identification of urinary infections associated to health care in NICU newborn patients. We must consider failure to thrive as the most common clinical manifestation for urinary infections in this age group.

Keywords: Neonatal urinary tract infection, bacteriological profile, nosocomial urinary infections.

o más a 5 leucocitos/campo en el examen general de orina (EGO). ${ }^{12}$

El objetivo del estudio es determinar el rendimiento diagnóstico del EGO frente al urocultivo en recién nacidos con sospecha de IVU hospitalizados en una UCIN, así como describir manifestaciones clínicas en este grupo de pacientes.

\section{MATERIAL Y MÉTODOS}

Estudio observacional y retrospectivo. Se incluyeron reportes de laboratorio de neonatos con sospecha clínica de IVU internados en la UCIN del Hospital Universitario «Dr. José Eleuterio González» de enero de 2018 a junio de 2019. Se definió a un neonato como un paciente menor a 30 días de vida. Se recolectaron características clínicas, parámetros bioquímicos del EGO y se compararon con el perfil bacteriológico del urocultivo.

Se consideraron con sospecha de IVU casos con datos clínicos o de laboratorio de infección. Se definió como IVU ante un urocultivo positivo tomado por cateterismo estéril. Se excluyeron recién nacidos con reingreso a la UCIN después de 10 días de su alta y que no contaran con alguno de los estudios de laboratorio. Este protocolo fue aprobado por el Comité de Ética en Investigación Institucional con clave de registro PE19-00024.

Consideramos positivos los parámetros del examen general de orina: leucocitos con recuento igual o mayor a 5 leucocitos/campo, esterasa leucocitaria presente, nitritos presentes, bacterias presentes, levaduras presentes.

Se reportaron las variables cuantitativas en términos de medidas de tendencia central y de dispersión, y las variables cualitativas en frecuencias y porcentajes. Se calcularon los valores de sensibilidad, especificidad, valor predictivo positivo y negativo por medio de tablas tetracóricas. Se consideró una $p<$ 0.05 como estadísticamente significativo. Los datos 
fueron analizados por medio del paquete IBM SPSS versión 25 (Armonk, NY: IBM Corp.).

\section{RESULTADOS}

Se incluyeron 172 pacientes con sospecha de IVU. El diagnóstico de IVU se confirmó en 32 (18.6\%) por medio del reporte de urocultivo positivo, 19 $(59.4 \%)$ mujeres. La edad media gestacional fue menor en pacientes con urocultivo positivo (33 \pm 4.5 vs $34.6 \pm 3.6$ semanas, $p=0.034)$. No encontramos diferencia en el género $(p=0.706)$ ni el peso medio al nacer de los recién nacidos $(2,099 \pm$ $953 \mathrm{~g}$ en pacientes con urocultivo positivo vs 2247 $\pm 798 \mathrm{~g}$ en los pacientes con urocultivo negativo, $\mathrm{p}=0.362$ ).

Las manifestaciones clínicas más frecuentes fueron: fiebre (45.3\%), falla de medro (27.3\%), apnea (16.9\%) e ictericia (10.4\%). La falla de medro se asoció con mayor frecuencia en recién nacidos con urocultivo positivo $(\mathrm{p}=0.006)$ (Tabla 1).

La incidencia de sepsis fue similar en ambos grupos, en pacientes con urocultivo positivo (31.2\%) y con urocultivo negativo (17.8\%) $(p=0.09)$, igual que la incidencia de meningitis $(6.2 \%$ y $2.1 \%$, respectivamente; $p=0.233$ ). Se presentó una defunción en cada grupo de pacientes ( $p=0.338)$.

De los 32 pacientes con urocultivos positivos se obtuvieron 38 aislamientos: Klebsiella pneumoniae (31.5\%), Escherichia coli (26.5\%), de los cuales $40 \%$ de estos fueron Escherichia coli productora de betalactamasas de espectro extendido (BLEE), Enterococcus faecium (10.5\%), Candida no albicans (8\%), Enterococcus faecalis (5.2\%), Staphylococcus coagulasa negativa (5.2\%), Pseudomonas aeruginosa (5.2\%), Enterobacter cloacae (2.6\%), Stenotrophomonas maltophilia (2.6\%), Acinetobacter baumannii (2.6\%).

Evaluamos el rendimiento diagnóstico de diferentes parámetros evaluados por el examen general de orina contra el resultado del urocultivo (Tabla 2). De los parámetros, la esterasa leucocitaria fue el parámetro que se asoció con mayor sensibilidad $(47.8 \%)$, y la combinación de leucocitos + esterasa leucocitaria con una sensibilidad en un $31.6 \%$. Los que presentaron una mayor especificidad fueron la presencia de levaduras (98.6\%) y de nitritos $(95.7 \%)$, y éstos dos últimos en combinación con leucocitos en orina tienen una especificidad de $99.3 \%$ y $98.6 \%$, respectivamente.

En los urocultivos positivos la esterasa leucocitaria se encontró una media de 70 (70-125) leucocitos/ $\mu \mathrm{L}$ y en los urocultivos negativos una media de 70 (15-125) leucocitos/ $\mu \mathrm{L}(p=0.138)$.

Las anormalidades estructurales urinarias fueron más frecuentes en pacientes con urocultivos positivos ( 25 vs $8.5 \%, p=0.009$ ). Los hallazgos de malformaciones en estos pacientes fueron: hidronefrosis grado III bilateral con ureterocele izquierdo e hidrocele moderado, hidronefrosis grado I bilateral con valvas uretrales posteriores, ectasia pielocalicial, dilatación pielocalicial, hipoplasia renal bilateral y quiste simple en corteza renal derecha.

\section{DISCUSIÓN}

La incidencia de IVU asociada con cuidados de la salud en la UCIN en nuestro periodo de estudio fue de $9.7 \%$. La prevalencia reportada por Sastre y colaboradores ${ }^{1}$ en un estudio de 301 neonatos ingresados a la UCIN fue del $1.1 \%$, y la reportada por Bauer y su equipo ${ }^{2}$ en 81 recién nacidos prematuros con muy bajo peso al nacer fue de $8.1 \%$.

Aunque en ambos grupos el predominio fueron pacientes prematuros, fue menor la edad gestacional en el grupo con urocultivos positivos (33 SDG), lo cual es considerado uno de los principales factores de riesgo para el desarrollo de IVU, en línea con lo reportado en la literatura, lo cual determina una mayor frecuencia de IVU neonatal en menores de 36

Tabla 1: Manifestaciones clínicas de pacientes con sospecha de infección urinaria y la relación con el resultado del urocultivo.

\begin{tabular}{lcccc} 
& $\begin{array}{c}\text { Global }(\mathrm{n}=172) \\
\mathrm{n}(\%)\end{array}$ & $\begin{array}{c}\text { Urocultivo positivo }(\mathrm{n}=32) \\
\mathrm{n}(\%)\end{array}$ & $\begin{array}{c}\text { Urocultivo negativo } \\
(\mathrm{n}=140) \mathrm{n}(\%)\end{array}$ & $p$ \\
\hline Fiebre & $78(45.3)$ & $11(34.4)$ & $67(47.9)$ & 0.167 \\
Falla de medro & $47(27.3)$ & $15(46.9)$ & $32(22.9)$ & 0.006 \\
Apnea & $29(16.9)$ & $2(6.3)$ & $27(19.3)$ & 0.076 \\
Ictericia & $18(10.4)$ & $4(12.5)$ & $14(10.0)$ & 0.749
\end{tabular}


Rev Latin Infect Pediatr. 2021; 34 (1): 22-26

\begin{tabular}{|c|c|c|}
\hline Criterio & Sensibilidad (\%) & Especificidad (\%) \\
\hline Leucocitos & 22.2 & 81.4 \\
\hline Esterasa leucocitaria & 47.8 & 63.1 \\
\hline Nitritos & 3.2 & 95.7 \\
\hline Bacterias & 10.7 & 80.6 \\
\hline Levaduras & 9.7 & 98.6 \\
\hline $\begin{array}{l}\text { Leucocitos + estera- } \\
\text { sa leucocitaria }\end{array}$ & 31.6 & 86.9 \\
\hline Leucocitos + nitritos & 3.2 & 98.6 \\
\hline $\begin{array}{l}\text { Leucocitos + } \\
\text { bacterias }\end{array}$ & 9.4 & 96.4 \\
\hline $\begin{array}{l}\text { Leucocitos + } \\
\text { levaduras }\end{array}$ & 8.3 & 99.3 \\
\hline
\end{tabular}

semanas de gestación según lo reporta Sastre JB y su grupo, ${ }^{1}$ donde en los neonatos con IVU nosocomial se encontró una media de $36.1 \pm 4.2(25-42)$ semanas de gestación.

El peso promedio de los pacientes con IVU fue de 2,099 g, no diferente al resto de los pacientes, aunque se ha descrito que, además de estar relacionado directamente con prematuridad, Barton $\mathrm{M}$ y colaboradores ${ }^{13}$ mencionan que aumenta el riesgo de IVU neonatal un 10\% con una relación inversa con el peso al nacer.

Se ha reportado en la literatura un predominio de IVU en el género masculino, por las características anatómicas de los recién nacidos; sin embargo, en nuestros pacientes fue ligeramente predominante el género femenino (59.4\%). ${ }^{14,15}$ En un estudio mexicano realizado por Peralta y su equipo ${ }^{8}$ encontraron un predominio en este género $(67.1 \%)$ en una cohorte de 70 pacientes.

Las manifestaciones clínicas de IVU en los neonatos son inespecíficas, en esta cohorte de neonatos hospitalizados con sospecha de IVU la fiebre fue un síntoma poco frecuente y en aquellos con IVU confirmada, la falla de medro fue la manifestación clínica reportada, estos hallazgos concuerdan con el estudio realizado por Movahedian y colaboradores, en el cual se reporta la ausencia de fiebre en $34.5 \%$ de los casos. ${ }^{6}$

Los pacientes con urocultivo positivo presentaron sepsis en un $31.2 \%$. Mohseny y su grupo ${ }^{16}$ encontraron que la tasa de sepsis en pacientes con IVU menores a 32 semanas de gestación fue de $11.3 \%$, por lo que sugiere realizar análisis de orina de forma rutinaria cuando se sospecha sepsis tardía en recién nacidos prematuros, como presunto factor de riesgo para infección. Downey LC y colaboradores ${ }^{17}$ de 1,162 neonatos con urocultivo positivo observaron concordancia en el $13 \%$ de los cultivos de sangre y el $3 \%$ de los cultivos de líquido cefalorraquídeo (LCR).

En este estudio, los gérmenes que predominaron en los urocultivos positivos fueron la $K$. pneumoniae con un $31.5 \%$, seguido de E. coli con un $26.5 \%$. Peralta RI y colaboradores ${ }^{8}$ reportaron con mayor frecuencia K. pneumoniae (44.3\%), y E. coli $(25.7 \%)$. Bauer y su equipo ${ }^{2}$ de 68 episodios de infección del tracto urinario en recién nacidos prematuros con muy bajo peso, el microorganismo más aislado fue K. pneumoniae (47\%), Enterobacter spp. (23\%), E. coli (13\%) y C. albicans (7.3\%). Vachharajani y su grupo de investigadores ${ }^{9}$ encontraron en neonatos prematuros hospitalizados con IVU E. coli (19\%), K. pneumoniae (19\%) seguido de Enterococcus (17\%).

Dentro de los urocultivos positivos para E. coli, el $40 \%$ fueron BLEE; un gran número de bacterias Gram negativas que causan infecciones urinarias nosocomiales producen BLEE y la mayoría son resistentes a múltiples fármacos. ${ }^{18}$

En la evaluación del rendimiento diagnóstico de los distintos parámetros del urianálisis y sus configuraciones, con el urocultivo, no encontramos una sensibilidad mayor al 50\%. Sin embargo, encontramos que algunos parámetros son altamente específicos, del $95.7 \%$ en la presencia de nitritos y $98.6 \%$ para presencia de levaduras. La especificidad de esterasa leucocitaria fue de $63.1 \%$, leucocitos $81.4 \%$ y bacterias de $80.6 \%$.

La tasa de malformaciones urinarias fue mayor en pacientes con urocultivo positivo ( $25 \mathrm{vs} 8.5 \%$ ), siendo un factor de riesgo de IVU. Como lo reportan Paschke y colaboradores y Lai y su equipo, ,319 la presencia de hidronefrosis leve en la mayor parte de los casos y en aproximadamente $5 \%$ de los pacientes se presenta hidronefrosis severa, riñón en herradura, agenesia renal unilateral, megauréter congénito.

\section{CONCLUSIONES}

En conclusión, en nuestro grupo de pacientes no encontramos un buen rendimiento diagnóstico para tamizaje bajo el uso de urianálisis; sin embargo, en pacientes con alta sospecha, algunos datos del laboratorio pudieran asociarse con la presencia de IVU, 
y anticipar el abordaje terapéutico antes de recibir el reporte del urocultivo. Los principales patógenos asociados con infecciones en esta población fueron K. pneumoniae y E. coli.

Demostramos la relación que existe entre factores de riesgo para el desarrollo de IVU nosocomial neonatal como lo es la prematurez, el peso bajo relacionado con la prematuridad y el género femenino. La falla de medro es una manifestación clínica para considerar infección de vías urinarias nosocomial. Es necesario considerar el urocultivo tomado por cateterismo estéril como el estándar de oro en infecciones urinarias en recién nacidos internados en la unidad neonatal.

\section{REFERENCIAS}

1. Sastre JB, Aparicio AR, Cotallo GD, Colomer BF, Hernández $M C$. Urinary tract infection in the newborn: clinical and radio imaging studies. Pediatr Nephrol. 2007; 22: 1735-1741.

2. Bauer S, Eliakim A, Pomearnz A, Regev R, Litmanovits I, Arnon $S$ et al. Urinary tract infection in very low birth weight preterm infants. Pediatr Infect Dis J. 2003; 22: 426-429.

3. Paschke AA, Zaoutis T, Conway PH, Xie D, Keren R. Previous antimicrobial exposure is associated with drugresistant urinary tract infections in children, Pediatrics. 2010; 125 (4): 664-672.

4. Falcão MC. Urinary tract infection in full-term newborn infants: risk factor analysis, Rev Hosp Clin Fac Med S Paulo. 2000; 55 (1): 9-16.

5. Mohammed D, EI Seifi O. Bacterial nosocomial infections in neonatal intensive care unit, Zagazig University Hospital, Egypt. Gaz Egypt Paediatr Assoc. 2014; 62: 72-79.

6. Movahedian AH, Mosayebi Z, Moniri R. Urinary tract infections in hospitalized newborns in Beheshti Hospital, Iran: a retrospective study. J Infect Dis Antimicrob Agents. 2007; 24 (1): 7-11.

7. García F, Nager A. Jaundice as an early sign of urinary tract infection in infancy. Pediatrics. 2002; 109 (5): 846-851.

8. Peralta RI, González CM, Pardo MR. Principales factores de riesgo asociados a infecciones nosocomiales de vías urinarias en un Servicio de Neonatología. Arch Inv Mat Inf. 2011; 3 (3): 138-144.

9. Vachharajani A, Vricella GJ, Najaf T, Coplen DE. Prevalence of upper urinary tract anomalies in hospitalized premature infants with urinary tract infection. J Perinatol. 2015; 35 (5): 362-366.
10. Kanellopoulos TA, Salakos C, Spiliopoulou I, Ellina A, Nikolakopoulou NM, Papanastasiou DA. First urinary tract infection in neonates, infants and young children: a comparative study. Pediatr Nephrol. 2006; 21: 11311137.

11. Ruangkit C, Satpute A, Vogt BA, Hoyen C, Viswanathan S. Incidence and risk factors of urinary tract infection in very low birth weight infants. J Neonatal Perinatal Med. 2016; 9 (1): 83-90.

12. Subcommittee on Urinary Tract Infection. Reaffirmation of AAP clinical practice guideline: the diagnosis and management of the initial urinary tract infection in febrile infants and young children 2-24 months of age. Pediatrics. 2016; 138 (6): e20163026.

13. Barton M, Bell Y, Thame M, Nicholson A, Trotman H. Urinary tract infection in neonates with serious bacterial infections admitted to the University Hospital of the West Indies. West Indian Med J. 2008; 57 (2): 101-105.

14. Arshad M, Seed PC. Urinary tract infections in the infant. Clin Perinatol. 2015; 42 (1): 17-28.

15. Arredondo GJ, Segura CE, Calderón JE, Mancilla RJ, Sánchez HG, Solórzano SF. Consenso mexicano en infecciones de vías urinarias en pediatría. Acta Pediatr Mex. 2007; 28 (6): 289-293.

16. Mohseny AB, van Velze V, Steggerda SJ, Smits-Wintjens VEHJ, Bekker V, Lopriore E. Late-onset sepsis due to urinary tract infection in very preterm neonates is not uncommon. Eur J Pediatr. 2018; 177 (1): 33-38.

17. Downey LC, Benjamin DK Jr, Clark RH, Watt KM, Hornik $\mathrm{CP}$, Laughon $\mathrm{MM}$ et al. Urinary tract infection concordance with positive blood and cerebrospinal fluid cultures in the neonatal intensive care unit. J Perinatol. 2013; 33 (4): 302306.

18. Rasool S, Mohsen E, Dariush S. Antimicrobial susceptibility pattern of extended-spectrum $\beta$-lactamase-producing bacteria causing nosocomial urinary tract infections in an Iranian referral teaching hospital. J Res Pharm Pract. 2014; 3 (1): 6-11.

19. Lai A, Rove K, Amin S, Vricella GJ, Coplen D. Diagnosis and management of urinary tract infections in premature and term infants. NeoReviews. 2018; 19 (6): e337-e348.

Correspondencia:

Dr. José Iván Castillo Bejarano

Av. Gonzalitos Núm. 235,

Mitras Centro, 64460,

Monterrey, Nuevo León, México.

Teléfono: $01818389-1111$, ext. 3355

E-mail: jicastillobejarano@gmail.com 